\title{
Translation and Transliteration
}

$\mathrm{T}$ ransliterating from Uzbek, a language that contains much borrowing from other tongues, into English, a language that similarly assimilates foreign words without difficulty, is no easy task-the results are inevitably imperfect. The system I have chosen attempts to create a consistent representation of the many unique cultural concepts that appear in the novel, while also maintaining accessibility for readers not familiar with Central Asia.

For words that have already been borrowed by English through other contexts - namely, Arabic, Persian, and South Asian languages - I maintain the commonly accepted English standard. For example, I write imam instead of imom, pilaf instead of palov or osh, and tandoor instead of tandir. Likewise, names that are known to English speakers through Arabic, Persian, and Turkish contexts, such as Nizami Ganjavi, receive the commonly accepted transliterations minus any diacritics. The larger Central Asian city names also receive their commonly accepted English transliterations. For the names of historical persons and places that are not well known, I often provide internationally accepted transliterations in the footnotes, while using their Uzbek transliteration in the text itself. For concepts specific to the sedentary Central Asian peoples, I transliterate names and those words that I gloss via the Uzbek Latin alphabet established in 1992 with some modifications.

I translate from the original 1936 edition of the text, which was printed in the short-lived 1930s Uzbek Latin alphabet, and from transliterated reproductions of that text. When Cho'lpon's novel was first republished in 1988 and in subsequent editions, the editors transliterated the original into the Uzbek Cyrillic alphabet, which was introduced in the 1940s. That transliteration erases some of the vowel harmony that the 1930s Uzbek literary language still possessed. The 1992 Latin alphabet obscures nothing when transliterating from 
Uzbek Cyrillic because each letter possesses a one-to-one transliteration equivalent. The 1992 alphabet in which transliterations appear presents only a few difficulties for the English reader: "o" is read as an ordinary English "o"; "o" is read as an open, almost full "a" sound; " $x$ " is read as a devoiced " $h$ " sound (often transliterated from other languages as " $k \mathrm{~h}$ "); "q" is a devoiced guttural velar; and " $\mathrm{g}$ " is the voiced pair of "q." Instead of using a different translation system for the several Russian names that appear here, I have decided to transliterate them through the Uzbek alphabet, the only caveats being that "b" becomes " $y$," and the soft sign ("b") becomes an apostrophe. I use common English equivalents for Russian names, such as Alexander for Aleksandr, only for the tsars and other figures well known in English.

I have modified the 1992 alphabet somewhat to ensure that transliterations in the text will not look unfamiliar to Middle Eastern specialists. The 1992 alphabet prescribes a forward apostrophe to indicate a glottal stop (the Arabic phoneme 'ayn). To distinguish them from the glottal stop, the Uzbek phonemes $o^{\prime}$ and $g^{\prime}$ are rendered with a backward apostrophe as $o^{\prime}$ and $g^{\prime}$. That backward apostrophe, however, looks similar to the turned comma ', which Middle Eastern specialists generally use to transliterate 'ayn. To avoid that confusion and maintain consistency with the rest of this translation series, this text uses the forward apostrophe for the Uzbek phonemes $o^{\prime}$ and $g^{\prime}$ and the turned comma for the glottal stop or 'ayn.

When citing Russian and Uzbek sources in the footnotes of the text, I use the Library of Congress transliteration system and the 1992 Uzbek Latin alphabet respectively. I do this to ease the burden for those who might look for these sources in a US library.

The glossary that follows the text of the novel provides definitions of lacunae found in the text. In the glossary I explain the terminology relating to rank and cultural customs of sedentary Central Asians that is important to understanding the social and cultural context of Cho'lpon's time. The first appearance of a glossed word in the text, with the exception of Sufi and Fitna, which are used as monikers, is marked in italics to indicate that the reader refer to the glossary. All subsequent uses of glossed words are unitalicized.

Regarding translation, I have tried to retain a feel of the original within the English text. Sometimes this means translating Uzbek-language idioms, of which Cho'lpon uses many, literally-rather than searching for rough equivalents in English. Where idioms or cultural realia require further explanation than given in the text, I indicate their meaning in the footnotes and glossary supplied at the end of the text. Likewise, I tried to maintain some of 
the syntax and word order that the author employs. That being said, clarity and rhythm often required that I break up Cho'lpon's frequent long sentences into smaller units.

Additionally, punctuation posed something of a challenge. Cho'lpon makes ample use of ellipses in the original text in ways that would confuse a contemporary English-language reader. He uses ellipses 1) to indicate a speaker is trailing off; 2) to indicate that something has been left unsaid by either the narrator or the characters (this is often sexual innuendo or the act of sex itself); 3 ) to indicate an impending contradiction, often at the end of chapters or sections, creating suspense; 4) to simulate stream of consciousness when the narrator enters the minds of characters; and 5) to denote the narrator's incomplete sentences, which are often used when describing the layout of a new space or room. I have retained ellipses in the first case and rarely in the cases of numbers two and four. 
